\title{
Avaliação psicológica dos cuidadores de mulheres com câncer pelo General Comfort Questionnaire ${ }^{1}$
}

\author{
Vera Lucia Rezende ${ }^{2}$ \\ Sophie Derchain \\ Neury José Botega \\ Luis Otávio Sarian \\ Daniela Landulfo Vial \\ Sirlei Siani Morais \\ Universidade Estadual de Campinas, Campinas-SP, Brasil \\ Antonio André Magoulas Perdicaris \\ Universidade Metropolitana de Santos, Santos-SP, Brasil
}

\begin{abstract}
Resumo: Este estudo avaliou o bem-estar global de 133 cuidadores de mulheres com câncer genital ou de mama, na fase avançada da doença, pelo General Comfort Questionnaire (GCQ) e analisou a associação com ansiedade, depressão, variáveis sociodemográficas e encargos do cuidador. Foi aplicada a Escala Hospitalar de Ansiedade e Depressão, o GCQ e uma entrevista semi-estruturada. Foram utilizadas a análise de variância (ANOVA), Tukey e a regressão linear múltipla em stepwise para determinar correlações entre as características do cuidador e bem-estar. O Alpha de Cronbach foi 0,8315. Segundo os resultados, quanto maior a idade do cuidador melhor o bem-estar $(\mathrm{p}<0,01)$. Cuidadores do sexo masculino apresentaram grau de bem-estar significativamente maior do que cuidadores do sexo feminino $(p=0,01)$. Maridos apresentaram grau de bem-estar significativamente maior do que todos os outros cuidadores $(p<0,001)$. Ansiedade $(p<0,01)$, depressão $(p=0,02)$ e ser mulher $(\mathrm{p}=0,01)$ foram fortemente relacionadas com menor bem-estar. O GCQ mostrou-se eficiente para realizar uma avaliação abrangente e multidimensional do cuidador.
\end{abstract}

Palavras-chave: psicologia, neoplasias, cuidadores, cuidados paliativos, ansiedade.

\section{Psychological assessment of the caregivers of women with cancer through the General Comfort Questionnaire}

\begin{abstract}
This study evaluated global well-being of 133 caregivers of women with genital or breast cancer in the advanced stage of disease through the General Comfort Questionnaire (GCQ) and evaluates the association with anxiety, depression, sociodemographic characteristics; and incumbencies of the main caregivers. The Hospital Anxiety and Depression Scale was completed, followed by the GCQ and an interview semi-structured. To assess the effects of the caregivers' characteristics on GCQ scores, analysis of variance followed by Tukey were applied. Stepwise multiple linear regressions were used. The Cronbach's Alpha coefficient was 0.8315 . Older caregiver presented better comfort $(\mathrm{p}<0.01)$. Caregivers male presented degree of well-being significantly higher than female caregivers. Patients' spouses showed significantly greater GCQ scores than other caregivers $(p<0.001)$. Anxiety $(p<0.01)$, depression $(p=0.02)$, and being woman $(p=0.01)$ were strongly associated with lower well-being. The GCQ was efficient to conduct a comprehensive and multidimensional assessment of the caregiver.
\end{abstract}

Keywords: psychology, neoplasms, caregivers, palliative care, anxiety.

\section{Evaluación psicologica de los cuidadores de mujeres con cáncer según el General Comfort Questionnaire}

Resumen: Este estudio evaluó el bienestar global de 133 cuidadores de mujeres con cáncer genital o de mama en etapas avanzadas de la enfermedad según el General Comfort Questionnaire (GCQ) y analizó la asociación con la ansiedad, depresión, variables sociodemográficas y responsabilidades del cuidador. Fue aplicada la Escala Hospitalaria de Ansiedad y Depresión, el GCQ y una entrevista semi-estructurada. Fueron utilizados el análisis de variación (ANOVA), Tukey y la regresión linear múltiple para determinar correlaciones posibles entre las características del cuidador y el bienestar. El Alpha de Cronbach fue 0.8315. Cuanto mayor la edad, mejor es el bienestar del cuidador $(\mathrm{p}<0.01)$. Cuidadores del sexo masculino mostraron mejor bienestar que cuidadores del sexo femenino $(\mathrm{p}=0.01)$. Los maridos presentaron grado de bienestar perceptiblemente mayor que los de todos los otros cuidadores $(p<0.001)$. La ansiedad $(p<0.01)$, la depresión $(p=0.02)$ y cuidadores del sexo femenino $(\mathrm{p}=0.01)$ estaban fuertemente relacionadas con poco bienestar. El GCQ reveló eficiencia para una evaluación global y multidimensional del cuidador.

Palabras clave: psicología, neoplasmas, cuidadores, cuidados paliativos, ansiedad.

\footnotetext{
1 Apoio: CAPES. Esse artigo é derivado da Tese de Doutorado defendida pela primeira autora, sob a orientação do segundo autor, juntos ao Programa de Pós-graduação da Faculdade de Ciências Médicas da Universidade Estadual de Campinas, Campinas, SP.

2 Endereço para correspondência:

Vera Lucia Rezende. Rua 2, 382. Condomínio Paineiras. CEP: 13.140-

000. Paulínia-SP, Brasil. E-mail: rezende.vera@gmail.com
} 
Desde a década de 1970, pacientes com câncer na fase avançada da doença, juntamente com seus familiares ou amigos, chamados aqui cuidadores informais, têm sido considerados como uma unidade e, cada vez mais, esses cuidadores formam uma variável significativa para a assistência (Rabow, Hauser, \& Adams, 2004). A Organização Mundial da Saúde considera o atendimento às necessidades dos cuidadores um dos principais objetivos dos cuidados paliativos e determina que se disponibilize um sistema de apoio para ajudar a família durante a doença do paciente e no processo do luto (World Health Organization [WHO], 1990).

A fase terminal é tida como a mais difícil e angustiante. Os principais problemas dos pacientes, geralmente, são administrar à dor, a insuficiência respiratória, a confusão, seguidos por ansiedade e depressão (Klinkenberg, Willems, Van der Wal, \& Deeg, 2004; Kovács, 2008). Cuidar de um familiar nesta fase representa desafios a ser superados, envolvendo longos períodos de tempo, desgastes físicos, custos financeiros, sobrecarga emocional, riscos mentais e físicos (Rabow e cols., 2004).

Estudos recentes demonstram que os cuidadores informais têm dificuldades para identificar o que o paciente sente. Suas percepções sobre os sentimentos e os sintomas do paciente correspondem mais à imaginação do que à realidade. Provavelmente muita angústia e conflitos poderiam ser minimizados mediante um bom atendimento ou uma comunicação mais eficaz (Lobchuk \& Vorauer, 2003). Os pacientes e seus cuidadores queixam-se da falta de informações sobre o manejo da dor, da fadiga e de como obter recursos para cuidados paliativos domiciliares. Um terço dos cuidadores expressa que, se houvesse disponibilidade, participariam de eventos educativos relacionados aos cuidados a serem ministrados aos pacientes na fase avançada da doença (Perdicaris, 2005). Outros aspectos estudados apontam para o despreparo dos familiares no enfrentamento da morte (Barbieri, Machado, \& Figueiredo, 2005)

Existe um inter-relacionamento entre sintomas psicológicos apresentados pelo paciente e pela família. A família é afetada pela doença e a dinâmica familiar afeta o paciente. Depressão no paciente pode desencadear depressão no cuidador, e vice-versa (Kurtz, Kurtz, Given, \& Given, 1995; Hodgson, Higginson, McDonnel, \& Butters, 1997). Desta forma, os cuidadores têm um papel muito importante nos aspectos práticos, sociais, físicos e emocionais do paciente, bem como nas decisões a serem tomadas no fim da vida (Armes \& Addington-Hall, 2003).

Nos estudos que abordam as conseqüências de cuidar na fase terminal da doença, a experiência é descrita como muito pesada e negativa (Klinkenberg e cols., 2004; Rezende, Derchain, Botega, \& Vial, 2005). Muitas vezes a depressão nesta fase é subestimada pela falta de conhecimento especializado das doenças psicológicas e pela visão equivocada de que é "normal" entre pacientes e seus familiares (Jones, 2001). Nesta fase ocorre fundamentalmente uma síndrome denominada "ansiedade familiar" que pode ser mais difícil de aliviar do que um problema físico ou dor (Higginson \& Priest, 1996).
A ansiedade familiar surge como conseqüência do enfrentamento da morte. Esta condição é uma das mais relevantes e parece mais preocupante do que a depressão. A depressão e a ansiedade familiar são muito freqüentes nas últimas quatro semanas antes do óbito em pacientes e seus cuidadores principais: um terço dos cuidadores apresenta ansiedade grave e este é o único item que se intensifica nas últimas semanas de vida do paciente (Hodgson e cols., 1997).

Além de poucos trabalhos examinarem pacientes e família ao mesmo tempo, a maioria avalia a família após a morte, no período do luto. Pouco se conhece sobre o estado psicológico do cuidador no período que antecede a morte do paciente (Higginson \& Priest, 1996; Hodgson e cols., 1997). Atualmente, surge a questão do que seria um "bom final de vida" para o paciente e qual seria o parâmetro de bem-estar global, inclusive para o cuidador (Nijboer, Triemstra, Tempelaar, Sanderman, \& van den Bos, 1999; Novak, Kolcaba, Steiner, \& Dowd, 2001).

A mensuração do impacto psicológico do cuidador é bastante complexa. Por esse motivo, na pesquisa científica utilizam-se as escalas. Sua finalidade é garantir que a informação coletada seja padronizada a fim de ser comparada de forma confiável (Deeken, Taylor, Mangan, Yabroff, \& Ingham, 2003).

\section{Instrumentos para avaliação dos cuidadores}

Um estudo de revisão analisou 471 artigos e selecionou apenas seis instrumentos específicos para avaliação da necessidade dos cuidadores de pacientes com câncer. Os resultados mostraram que nenhum instrumento era completo em relação a todas as dimensões dos cuidados paliativos: sintomas físicos, sintomas psicológicos, suporte social, necessidades econômicas, expectativas e esperanças, crenças existenciais e espirituais (Osse e cols., 2000). De acordo com Nijboer e cols. (1999) muitos instrumentos avaliam apenas o estado físico, emocional, social e atividade diária.

Atualmente, reconhece-se a necessidade de realizar uma avaliação global do cuidador que aborde diferentes tipos de dimensões. Para a escolha de um instrumento adequado é necessário realizar um estudo cuidadoso embasado em três considerações: aplicabilidade para uma população-alvo específica; multidimensionalidade que inclua aspectos positivos e negativos, objetivos e subjetivos; e boa propriedade psicométrica, ou seja, a capacidade de mensuração psicológica (Francke, 2000; Deeken e cols., 2003).

O General Comfort Questionnaire (GCQ), recentemente adaptado para pacientes na fase avançada da doença e seus cuidadores informais, publicado por Novak e cols. (2001) e traduzido para o português (Rezende e cols., 2005) foi selecionado e utilizado neste estudo por permitir uma avaliação global do bem-estar dos cuidadores no fim da vida do paciente. Tem como referência o conceito de "bem-estar" definido através de múltiplos indicadores do bem-estar global: físico, ambiental, social, psicológico e espiritual. O GCQ oferece a oportunidade de ampliar o campo de pesquisa na área de 
cuidados paliativos e propõe-se a avaliar tanto os aspectos negativos como os positivos do cuidar (Novak e cols., 2001).

O objetivo deste trabalho foi avaliar o bem-estar global dos cuidadores informais de pacientes com câncer ginecológico ou de mama na fase avançada da doença em um hospital-escola brasileiro de referência para o tratamento de câncer e verificar a associação com ansiedade, depressão, variáveis sociodemográficas e encargos do cuidador principal.

\section{Método}

\section{Participantes}

Para este estudo descritivo exploratório de corte transversal foram entrevistados 133 cuidadores principais, indicados espontaneamente por pacientes acometidas por câncer genital (62) ou câncer de mama (71) em fase terminal, e acamadas por mais de $50 \%$ das horas de vigília ou incapacitadas.

\section{Instrumentos}

O General Comfort Questionnaire (GCQ) foi utilizado em sua versão em português para o cuidador (Rezende e cols., 2005). Foi testado anteriormente por Kolcaba e Fox (1999) nos Estados Unidos em 51 pacientes e em seus cuidadores e apresentou forte fidedignidade e segurança, com índice Alpha de Cronbach igual a 0,97, evidenciando ótima propriedade psicométrica. Contém 49 perguntas, cujas respostas vão de um a seis. Para a aplicação é aconselhada a utilização de um cartão, do tamanho de uma folha de papel sulfite, aproximadamente, contendo a graduação $(1,2,3,4$, 5 e 6), a fim de que o cuidador visualize e escolha um valor para cada questão apresentada.

A Escala Hospitalar de Ansiedade e Depressão (Hospital Anxiety and Depression Scale, HAD foi validada no Brasil, mostrou-se de fácil compreensão com capacidade de revelar casos de transtornos de humor (Botega, Bio, Zomignani, Garcia, \& Pereira, 1995). Seu uso no meio oncológico já é bastante reconhecido. De acordo com o Grupo Internacional de Consenso sobre depressão e ansiedade a escala HAD é um instrumento capaz de medir a ansiedade e a depressão separadamente, tendo sido usada com sucesso para avaliar sintomas psicológicos tanto em pacientes com outras doenças instaladas como em indivíduos não considerados doentes (Ballenger e cols., 2001). É um instrumento de autopreenchimento que possui 14 itens, dos quais sete pesquisam ansiedade (HAD-A) e sete pesquisam depressão (HAD-D) (Zigmond \& Snaith, 1983).

\section{Procedimento de coleta de dados}

$\mathrm{O}$ estudo foi aprovado pelo Comitê de Ética em Pesquisa da Faculdade de Ciências Médicas da Universidade Estadual de Campinas. Participaram da pesquisa somente os cuidadores que firmaram o Termo de Consentimento Livre e Esclarecido. Foram assegurados o sigilo e o uso exclusivo das informações no estudo, assim como o direito de não participação ou de interrupção a qualquer momento. Todos os princípios éticos contidos na última versão da
Declaração de Helsinki (World Medical Association) foram respeitados e cumpridos.

Os dados foram coletados entre agosto de 2002 a maio de 2004 na enfermaria de oncologia do Centro de Atenção Integral à Saúde da Mulher - CAISM. Inicialmente, foi realizada uma breve entrevista com a paciente para que ela indicasse o seu cuidador principal. Quando apresentava mais do que um, era solicitado que indicasse apenas um, aquele que era mais significativo. Em seguida, o cuidador foi contatado.

Os instrumentos foram lidos em voz alta pelo entrevistador para contornar eventuais problemas de analfabetismo. Novak e cols. (2001) não recomendam o autopreenchimento do GCQ, em função dos participantes estarem experienciando a morte iminente de um ente querido.

Inicialmente foi aplicada a escala HAD, seguida da GCQ, e após foi realizada uma entrevista semi-estruturada visando à complementação de informações (dados de identificação, se mora junto com a paciente, se é acompanhante no hospital, se cuida de outras pessoas e se existe rodízio familiar). Os cuidadores foram entrevistados por duas psicólogas, na sala de atendimento psicológico ou na sala de espera da enfermaria.

\section{Procedimento de análise dos dados}

O escore final do GCQ é construído como uma escala começando com 49 pontos, péssimo bem-estar, e finalizando com 294 pontos, excelente bem-estar (Novak e cols., 2001). $\mathrm{O}$ questionário inclui perguntas redigidas nas formas positiva e negativa e a pontuação nos itens redigidos na forma negativa foi invertida após a aplicação do instrumento, ou seja, quando apresentava a pontuação seis era convertida para um, e assim por diante.

Cada item do HAD tem uma gradação de 0-3; a soma dos itens fornece a pontuação total (de 0 a 21 , em cada subescala). Uma pontuação de oito ou mais, tanto na subescala de ansiedade quanto na de depressão, foi considerada neste estudo sugestiva de transtorno de ansiedade e depressão, respectivamente (Botega, Pondé, Medeiros, Lima, \& Guerreiro, 1998).

O valor médio e o desvio padrão (DP), valores mínimos e máximos, foram calculados para cada questão no GCQ. O coeficiente Alpha de Cronbach foi usado para testar a consistência interna do GCQ. Para avaliar a associação das características dos cuidadores com os resultados obtidos foi realizada a análise de variância (ANOVA) seguida de Tukey, ou t de Student, e os valores de $p$ foram calculados para cada variável estudada. Para verificar a correlação entre sintoma de ansiedade e depressão com os resultados do GCQ foi utilizado o coeficiente de correlação de Pearson. Em seguida foi aplicada uma regressão linear multivariada em stepwise para verificar quais variáveis dos cuidadores estão mais associadas com o bem-estar avaliado pelo GCQ. Os critérios de seleção das variáveis foram baseados na análise estatística de Cp Mallow e R (Altman, 1991; SAS Institute, 1999/2001). 


\section{Resultados}

$\mathrm{O}$ valor médio das questões variou de 1,1 a 5,8 . Os maiores e menores valores médios das questões do GCQ

encontram-se nas Tabelas 1 e 2, respectivamente, bem como o desvio padrão (DP) e as variações individuais de cada resposta. O Alpha de Cronbach foi 0,8315 .

Tabela 1

Maiores escores médios obtidos com o GCQ

\begin{tabular}{|c|c|c|c|c|c|}
\hline Questão & Maiores escores médios & Média & $( \pm \mathbf{D P})$ & Mínimo t & Máximo \\
\hline 23 & Eu gosto que o quarto dela esteja tranqüilo & 5,8 & 0,7 & 2 & 6 \\
\hline 38 & Nós estamos bem em nosso Relacionamento & 5,8 & 0,5 & 2 & 6 \\
\hline 8 & Eu sei que sou amado & 5,8 & 0,7 & 1 & 6 \\
\hline 49 & Deus está me ajudando & 5,8 & 0,6 & 1 & 6 \\
\hline 47 & Ela está limpa e seca & 5,7 & 1,0 & 1 & 6 \\
\hline 33 & Eu me sinto suficientemente forte para fazer algumas coisas para ela & 5,6 & 0,5 & 1 & 6 \\
\hline 31 & Quando olho para trás vejo que vivemos uma boa vida & 5,6 & 1,0 & 1 & 6 \\
\hline 18 & Eu sou capaz de me comunicar com quem eu amo & 5,5 & 1,2 & 1 & 6 \\
\hline 21 & Eu tenho pessoas especiais que fazem eu me sentir apoiada & 5,5 & 1,1 & 1 & 6 \\
\hline 35 & Eu me sinto confiante espiritualmente & 5,5 & 1,0 & 1 & 6 \\
\hline 5 & Minha crença me traz paz de espírito & 5,4 & 1,2 & 1 & 6 \\
\hline 15 & Eu me sinto culpado* & 5,2 & 1,6 & 1 & 6 \\
\hline 30 & Essa situação me inspira & 5,2 & 1,5 & 1 & 6 \\
\hline 27 & Eu posso crescer com essa situação & 5,2 & 1,4 & 1 & 6 \\
\hline 7 & Minha vida não vale a pena nesse momento* & 5,1 & 1,5 & 1 & 6 \\
\hline 25 & A temperatura deste quarto é agradável & 5,0 & 1,5 & 1 & 6 \\
\hline 3 & Quando eu preciso de ajuda tenho a quem recorrer & 5,0 & 1,5 & 1 & 6 \\
\hline 6 & As enfermeiras me trazem esperança & 5,0 & 5,0 & 1 & 6 \\
\hline 44 & Os amigos se lembram de nós através de recados e telefonemas & 5,0 & 1,6 & 1 & 6 \\
\hline
\end{tabular}

(133 cuidadores informais)

*Invertidas

Tabela 2

Menores escores médios obtidos com o GCQ

\begin{tabular}{|c|c|c|c|c|c|}
\hline Questão & Menores escores médios & Média & $(+\mathrm{DP})$ & Mínimo t & Máximo \\
\hline 4 & Eu me preocupo com minha família* & 1,1 & 1,1 & 1 & 6 \\
\hline 34 & Eu penso no desconforto dela constantemente* & 1,5 & 1,3 & 1 & 6 \\
\hline 45 & O estado emocional dela me deixa triste* & 1,6 & 1,1 & 1 & 6 \\
\hline 36 & $\begin{array}{l}\text { Eu tenho necessidade de ter mais informações } \\
\text { sobre a condição dela* }\end{array}$ & 2,0 & 1,7 & 1 & 6 \\
\hline 24 & Gostaria de ter mais contato com o médico* & 2,0 & 1,7 & 1 & 6 \\
\hline 48 & Tenho preocupações com a parte financeira* & 2,7 & 1,9 & 1 & 6 \\
\hline 46 & Eu penso muito no futuro* & 1,6 & 1,3 & 1 & 6 \\
\hline 12 & É difícil suportar a dor emocional que eu sinto* & 2,5 & 1,8 & 1 & 6 \\
\hline 22 & $\begin{array}{l}\text { Eu tenho passado por mudanças que me } \\
\text { deixam incomodado* }\end{array}$ & 2,5 & 2,5 & 1 & 6 \\
\hline 20 & Eu sinto medo do que vem pela frente* & 2,7 & 1,9 & 1 & 6 \\
\hline 28 & Esse ambiente é depressivo* & 3,0 & 2,0 & 1 & 6 \\
\hline 10 & Eu tenho dificuldade para descansar* & 3,0 & 1,8 & 1 & 6 \\
\hline
\end{tabular}

*Invertidas 
A Tabela 3 contém as características sociodemográficas e clínicas dos cuidadores principais associadas aos escores médios obtidos com o GCQ. A maioria dos cuidadores tinha entre 36 e 55 anos $(42,3 \%)$ e apenas $20,0 \%$ tinham mais de 55 anos de idade. Cuidadores mulheres $(67,7 \%)$ foram mais freqüentes que os do sexo oposto e a quase maioria relatou possuir uma religião. $\mathrm{O}$ grau de parentesco mais freqüente com a paciente foi o de filha $(43,1 \%)$. Trinta e um $(23,8 \%)$ cuidadores foram os maridos. A maioria dos cuidadores era casada $(64,6 \%)$ e residia junto com a paciente $(71,5 \%)$. As proporções de cuidadores desempregados $(39,2 \%)$ e aposentados $(20,0 \%)$ foram relativamente altas. Os cuidadores tinham com quem revezar (rodízio) em 65,4\% dos casos estudados; no entanto $46,2 \%$ desses cuidavam também de outras pessoas além da paciente. A maioria $(83,1 \%)$ referia mudanças em suas rotinas em função do cuidar. Sintomas de ansiedade foram detectados em 75,3\% dos cuidadores e sintomas de depressão em $53,8 \%$.

Tabela 3

Características sociodemograficas e clínicas dos cuidadores, escores médios e desvio-padrão do GCQ

\begin{tabular}{|c|c|c|c|}
\hline Caracteristicas & $\mathbf{N}(\%)$ & Media (DP) & $\mathbf{p}$ \\
\hline \multicolumn{4}{|l|}{ Idade } \\
\hline$<35$ & $49(37,7)$ & $197,5(23,8)$ & \\
\hline $36-55$ & $55(42,3)$ & $201,2(24,2)$ & \\
\hline$\geq=56$ & $26(20,0)$ & $210,8(26,6)$ & $<0,01$ \\
\hline \multicolumn{4}{|l|}{ Sexo } \\
\hline Feminino & $88(67,7)$ & $198(25,2)$ & \\
\hline Masculino & $42(33,3)$ & $209,6(22,2)$ & 0,01 \\
\hline \multicolumn{4}{|l|}{ Religião } \\
\hline Não & $8(6,1)$ & $191,1(30,1)$ & \\
\hline Sim & $122(93,9)$ & $202,4(24,4$ & 0,2134 \\
\hline \multicolumn{4}{|l|}{ Parentesco } \\
\hline Filha & $56(43,1)$ & $196,2(25,8)$ & \\
\hline Marido & $31(23,8)$ & $214,0(22,3)$ & $<0,001$ \\
\hline Outros* & $43(33,1)$ & $200,1(22,7)$ & \\
\hline \multicolumn{4}{|l|}{ Estado civil } \\
\hline Casado & $84(64,6)$ & $204,1(24,3)$ & \\
\hline Outros $* *$ & $46(35,4)$ & $197,5(25,5)$ & 0,14 \\
\hline \multicolumn{4}{|l|}{ Mora com a paciente } \\
\hline Não & $37(28,5)$ & $198,8(22,8)$ & \\
\hline Sim & $93(71,5)$ & $202,9(25,6)$ & 0,39 \\
\hline \multicolumn{4}{|l|}{ Ocupação } \\
\hline Empregado & $53(40,8)$ & $203,5(22,2)$ & \\
\hline Não empregado*** & $51(39,2)$ & $196,1(26,0)$ & 0,07 \\
\hline Aposentado & $26(20,0)$ & $209,2(26,2)$ & \\
\hline \multicolumn{4}{|l|}{ Tempo entre a entrevista e o óbito } \\
\hline$<30$ dias & $69(53,1)$ & $201,7(24,2)$ & \\
\hline$>30$ dias & $61(46,9)$ & $201,8(25,6)$ & 0,98 \\
\hline \multicolumn{4}{|l|}{ Rodízio } \\
\hline Não & $45(34,6)$ & $201,0(27,0)$ & \\
\hline Sim & $85(65,4)$ & $202,1(23,7)$ & 0,80 \\
\hline \multicolumn{4}{|l|}{ Cuida de outras pessoas } \\
\hline Não & $70(53,8)$ & $205,6(24,9)$ & \\
\hline Sim & $60(46,2)$ & $197,2(24,1)$ & 0,06 \\
\hline \multicolumn{4}{|l|}{ Rotina mudou } \\
\hline Não & $22(16,9)$ & $210(25,9)$ & \\
\hline $\operatorname{Sim}$ & $108(83,1)$ & $200,0(24,5)$ & 0,07 \\
\hline \multicolumn{4}{|l|}{ Ansiedade $* * * *$} \\
\hline Sim & $98(75,3)$ & $195,9(23,6)$ & \\
\hline Não & $32(24,7)$ & $219,7(19,7)$ & $<0,001$ \\
\hline \multicolumn{4}{|l|}{ Depressão } \\
\hline Sim & $70(53,8)$ & $193,8(24,9)$ & \\
\hline Não & $60(46,2)$ & $211,0(21,5)$ & $<0,001$ \\
\hline
\end{tabular}

* filho (9), irmã (9), mãe (9), vários (17); ** solteiro (29), divorciado (8), viúvo (10); *** dona de casa (28), estudante (8), desempregado (24); DP = Desvio Padrão; **** Ansiedade e depressão foram definidas com escore $\geq=8$ na escala HADS 
A análise univariada revelou que apenas algumas características associavam-se com o bem-estar global. O bem-estar foi significativamente associado com a faixa etária. Quanto mais novo o cuidador, pior o seu bem-estar. Cuidadores do sexo masculino apresentaram grau de bem-estar significativamente maior do que cuidadores do sexo feminino $(p=0,01)$. Maridos apresentaram grau de bem-estar significativamente maior do que todos os outros cuidadores $(p<0,001)$. Sintomas de ansiedade e depressão também foram fortemente associados com baixos valores no GCQ ( $p<0,001$ para ambos). A contagem média do GCQ para os 98 cuidadores que revelaram sintomas de ansiedade foi 195,9 $(\mathrm{DP}=23,6)$, comparado com 219,7 $(\mathrm{DP}=19,7)$ para os cuidadores sem esses sintomas. Do mesmo modo, sintomas de depressão associaram-se fortemente a menores níveis de bem-estar. Cuidadores que tinham sintomas de depressão tiveram a contagem média do GCQ de 193,8 (DP=24,9) comparada com 211,0 $(\mathrm{DP}=21,5)$ do grupo sem sintomas.

$\mathrm{Na}$ Tabela 4 encontram-se os resultados da análise multivariada com seleção em stepwise. De todas as características dos cuidadores estudadas, apenas três estavam fortemente relacionadas com baixo nível de bem-estar global: presença de ansiedade $(\mathrm{p}<0,01)$, de depressão $(p=0,02)$ e o fato de o cuidador ser mulher $(p=0,01)$.

Tabela 4

Características do cuidador que afetam negativamente o bem-estar global após regressão linear multivariada

\begin{tabular}{lccccc}
\hline \multicolumn{1}{c}{ Características } & Parâmetro & SE & p & R2 & Cp \\
\hline Ansiedade & $-16,907$ & 6,851 & $<0,01$ & 0,236 & 9,122 \\
Ser Mulher & $-13,051$ & 6,382 & 0,01 & 0,312 & 3,970 \\
Depressão & $-14,425$ & 6,105 & 0,02 & 0,362 & 1,228 \\
Final & - & - & $<0,01$ & 0,362 & 1,212 \\
\hline
\end{tabular}

\section{Discussão}

A avaliação do cuidador identificou aspectos negativos associados com a extenuante demanda do cuidar e aspectos positivos, que são bem menos conhecidos.

\section{O impacto negativo no bem-estar global do cuidador}

Mais de 70\% dos cuidadores apresentavam ansiedade e mais de $50 \%$ depressão e constatou-se que a presença desses sintomas exerce um efeito negativo sobre o bem-estar global do cuidador. Em estudos anteriores esses índices são bem menores (Carter \& Chang, 2000) e já inspiram grande preocupação. A ansiedade no cuidador pode ser severa durante a fase de cuidados paliativos e tende a se agravar na última semana de vida, sendo muito difícil de ser quantificada e aliviada (Calson, Ottenbreit, St. Pierre, \& Bultz, 2001; Higginson \& Priest, 1996). A mensuração desses sintomas psicológicos revelou o quanto, no final da vida do paciente, o cuidador está desgastado e com a saúde mental comprometida, sendo que a "ansiedade familiar" foi registrada em um número admirável de cuidadores, superior ao encontrado por Higginson e Priest (1996). Esses dados levam a pensar em medidas preventivas urgentes, pois a ansiedade, depois de instalada é muito difícil de ser eliminada.

Os cuidadores que não apresentaram ansiedade ou depressão tiveram níveis elevados de bem-estar global em praticamente todos os domínios: social, psicoespiritual, ambiental e físico. A integridade da saúde mental do cuidador contribuiu consideravelmente para o seu bem-estar global. Tais resultados apontam para uma equiparação negativa entre as duas escalas HAD (para ansiedade e depressão) e GCQ (para bem-estar global), apoiada nos valores estatisticamente significativos obtidos da relação entre elas.
O fato de o cuidador ser mulher mostrou-se fortemente relacionado com o baixo nível de bem-estar global. Uma possível razão é que quando a mulher assume cuidar de uma paciente com câncer de mama ou genital, confronta-se a todo instante com a possibilidade de também adoecer, o que é muito desgastante emocionalmente (Cella \& Bonomi, 1995; Kessler, 1989). Os cuidadores vivenciam temores e fantasias relacionadas à herança genética do câncer, imaginam que também poderão desenvolver a doença, adicionando ansiedade a esta população (Erblich, Bovbjerg, \& Valdimarsdottir, 2000; Silva, 2003). Nesse sentido, uma atenção especial aos cuidadores do sexo feminino, especialmente as filhas, necessita ser praticada, pois é a população mais freqüente e vulnerável conforme os achados revelados por esse estudo.

O fato de os homens se saírem melhor do que as mulheres, em termos de bem-estar no ambiente hospitalar, foi um resultado singular. É mais comum observar e estudar a mulher no papel de cuidadora. No entanto, quando o homem assume esse papel e entre eles o marido, está propenso a ter maior aceitação, pois o casal imaginava poder vir a ser o cuidador um do outro, no caso de uma doença (Kristjanson, Leis, Koop, Carrière, \& Mueller, 1997)

As maiores preocupações antes do óbito, que mais contribuíram para baixar o bem-estar global dos cuidadores, foram: a família (como irá reagir frente à perda?), a dor física e emocional da paciente (ela está desconfortável, triste, angustiada?). De acordo com Kurtz e cols. (1995) e Parkes (1998), tristeza e depressão nos pacientes desencadeiam problemas psicológicos em suas famílias e vice-versa. $\mathrm{O}$ inter-relacionamento entre as necessidades do paciente e da família, bem como entre os sintomas psicológicos de ambos, foram amplamente estudados por Hodgson e cols. (1997). No presente estudo confirmou-se essa intensa troca emocional, 
evidenciando o quanto as preocupações com o bem-estar do paciente afetaram o bem-estar do cuidador.

Em seguida, identificamos que a falta de informações sobre a condição da paciente foi o fator que mais pesou para o declínio do bem-estar global do cuidador. Estudos anteriores já apontavam que os cuidadores têm necessidade de uma boa comunicação com a equipe de saúde e de obter o máximo de informações sobre o tratamento e os cuidados específicos no fim da vida (Perdicaris, 2005). Silva (2003) enfatiza que a falta de informação e a imaturidade dos profissionais para lidar com pacientes gravemente enfermos são os maiores problemas e um dos principais geradores de ansiedade familiar no âmbito hospitalar. Uma doença com longa duração, como é o câncer, tem resultados ainda piores, pois essas lacunas vão se ampliando e prejudicam até o processo de elaboração do luto (Butler e cols., 2005). No entanto, informar adequadamente não é uma simples questão de mudança nos padrões de comunicação, o que, de acordo com Carvalho (2003), é um dos maiores desafios para as equipes de cuidados paliativos, pois em sua base encontram-se a exposição à morte, que é sempre uma experiência traumática. Por isso é imprescindível cuidar também da equipe e garantir condições favoráveis de trabalho para lidarem adequadamente com questões tão complexas. Acreditamos que somente assim poderão desenvolver uma boa comunicação da informação, orientação e dos cuidados necessários ao paciente.

\section{O impacto positivo no bem-estar global do cuidador}

Os instrumentos utilizados permitiram quantificar sintomas psicológicos e identificar, além dos aspectos negativos, os aspectos positivos e subjetivos que favorecem o bem-estar. De acordo com Nijboer e cols. (1999) e Novak e cols. (2001), essa área é bem menos conhecida e raro são os trabalhos que avaliam esses fatores.

O que mais contribuiu para a elevação do bem-estar global foi o cuidador constatar que o quarto da paciente estava tranqüilo. Os efeitos do ambiente no bem-estar do cuidador ainda são pouco estudados (Nijboer e cols., 1999). Esses achados evidenciam o quanto esses fatores são importantes e que vale a pena investir na melhora do ambiente hospitalar. Muitas vezes são medidas simples, concretas e rápidas que tornam um ambiente mais tranqüilo e acolhedor: o respeito ao silêncio, uma iluminação indireta, uma adequada escolha de cores, boa ventilação, limpeza, dentre outras. A atmosfera ambiental reflete um tipo de acolhimento, especialmente útil para quem já está muito cansado, sensível a estímulos desagradáveis do ambiente.

O bom relacionamento entre cuidador e paciente, aliado ao fato de o cuidador sentir-se amado e ajudado por Deus elevaram o seu bem-estar. Essas questões tiveram igualmente altíssimos escores. Esses são aspectos subjetivos que puderam ser quantificados em um número significativo de cuidadores. De acordo com Kaasa e Loge (2003), no final da vida a espiritualidade e as questões existenciais tornam-se proeminentes, mas na literatura ainda são escassos os trabalhos que avaliam essa dimensão (Deeken e cols., 2003). A implantação de uma abordagem psicológica que contemple a dimensão espiritual na prática dos cuidados paliativos ainda é pouco aplicada e reconhecida. Para Gimenes (2003), os profissionais devem romper com o reducionismo proveniente do conhecimento exclusivamente racional e científico e resgatar uma visão de homem mais global e transcendente, e propõe a integração entre ciência e espiritualidade.

\section{Considerações finais}

O GCQ é um instrumento abrangente e sua utilização veio ao encontro da necessidade de realizar uma avaliação multidimensional. É um dos raros instrumentos que inclui questões relacionadas com a espiritualidade e o meio ambiente.

O Alpha de Cronbach indicou que a escala é adequada, possuindo uma excelente consistência interna. O GCQ foi utilizado na versão para o cuidador, pela primeira vez no Brasil neste estudo. Embora seja um instrumento longo, pode ser aplicado por qualquer profissional da área da saúde. Ressalta-se, no entanto, que sua análise final é complexa e demanda conhecimento especializado nesta área.

Sua utilização permite identificar diversos fatores que diminuem ou elevam o bem-estar global do cuidador. As preocupações com a família, com o desconforto da paciente e o seu estado emocional são os fatores que mais desfavorecem o seu bem-estar. $\mathrm{O}$ fato de o quarto da paciente estar tranqüilo, o bom relacionamento com ela e sentir-se amado são alguns dos principais fatores que mais o elevam.

Esses conhecimentos permitem que medidas preventivas e ajustes pontuais possam ser introduzidos antes da ocorrência do óbito da paciente, para minimizar o sofrimento intenso nesta fase. Esses achados poderão servir de base para outros estudos que visem à avaliação dos cuidadores de pacientes com câncer na fase avançada da doença.

Algumas considerações acerca do método utilizado por este trabalho fazem-se necessárias. Por se tratar de um estudo transversal, não podemos avaliar a trajetória dinâmica do cuidador. No entanto, em razão da escassez de estudos com cuidadores na fase avançada da doença utilizando instrumentos padronizados específicos, uma investigação preliminar foi necessária para produzir informações sobre os cuidadores. Outro problema que pode surgir com um estudo do tipo transversal é a possibilidade do viés de seleção. O critério adotado foi rigorosamente seguido, ou seja, todas as pacientes, no período de coleta de dados estabelecido, foram entrevistadas e a eleição do cuidador principal foi feita pela paciente, e nos casos em que ela se apresentava confusa, teve ajuda de um familiar para realizá-la, sem a intervenção dos pesquisadores. Este cuidado evitou que tivéssemos uma amostra tendenciosa. Mesmo assim, trata-se de um grupo não representativo, pois faz referência a um centro terciário especializado. Estudos adicionais envolvendo número adequado de pessoas seriam necessários para uma futura validação do GCQ em sua versão em língua portuguesa e sua aplicação na população brasileira.

Uma importante limitação do presente trabalho foi a avaliação apenas do cuidador e não da díade cuidador-paciente. 
A sugestão para futuros estudos é a análise da qualidade do vínculo entre cuidadores e pacientes. Notadamente, é importante reafirmar a relevância que teve neste estudo quando o marido foi o cuidador.

\section{Referências}

Altman, D. G. (1991). Pratical statistics for medical research. London: Chapman and Hall.

Armes, P. J., \& Addington-Hall, J. M. (2003). Perspectives on symptom control in patients receiving community palliative care. Palliative Medicine, 17, 608-615.

Ballenger, C. J., Davidson, J. R. T., Lecrubier, Y., Nutt, D. J., Jones, R. D., \& Berard, R. M. F. (2001). Consensus statement on depression, anxiety and oncology. The Journal of Clinical Psychiatry, 62(supl. 8), 64-67.

Barbieri, A. L., Machado, A. A., \& Figueiredo, M. A. C. (2005). Estudo qualitativo de crenças relativas aos cuidados domiciliares e à morte entre familiares de portadores de HIV/AIDS e câncer. Paidéia (Ribeirão Preto), 15, 427-432.

Botega, N. J., Ponde, M. P., Medeiros, P., Lima, M. G., \& Guerreiro, C. A. M. (1998). Validação da escala hospitalar de ansiedade e depressão (HAD) em pacientes epiléticos ambulatoriais. Jornal Brasileiro de Psiquiatria, 47, 285-289.

Botega, N. J., Bio, M. R., Zomignani, M. A., Garcia Jr., C., \& Pereira, W. A. B. (1995). Transtornos do humor em enfermaria de clínica médica e validação de escala de medida (HAD) de ansiedade e depressão. Revista de Saúde Pública, 29, 355-363.

Butler, L. D., Field, N. P., Bush, A. L., Seplaki, J. E., Hastings, T. A., \& Spiegel, D. (2005). Anticipating loss and other temporal stressors predict traumatic stress symptoms among partners of metastatic/recurrent breast cancer patients. Psychooncology, 14, 492-502.

Calson, L. E., Ottenbreit, N., St. Pierre, M., \& Bultz, B. D. (2001). Partner understanding of the breast and prostate cancer experience. Cancer Nursing, 24, 231-239.

Carter, P.A., \& Chang, B. L. (2000). Sleep and depression in cancer caregivers. Cancer Nursing, 23, 410-415.

Carvalho, V. A. (2003). Cuidados com o cuidador. O Mundo da Saúde, 27, 138-146.

Cella, D.F., \& Bonomi, A. E. (1995). Measuring quality of life: 1995 update. Oncology, 9(suppl. 11), 47-60.

Deeken, J. F., Taylor, K. L., Mangan, P., Yabroff, K. R., \& Ingham, J. M. (2003). Care for the caregivers: A review of self-report instruments developed to measure the burden, needs, and quality of life of informal caregivers. Journal of Pain and Symptom Managemen, 26, 922-953.

Erblich, J., Bovbjerg, D. H., \& Valdimarsdottir, H. B. (2000). Looking forward and back: Distress among women at familial risk for breast cancer. Annals of Behavioral Medicine, 22, 53-59.

Francke, A. L. (2000). Evaluative research on palliative support teams: A literature review. Patient Education and Counseling, 41,83-91.
Gimenes, M. G. G. (2003). A passagem entre a vida e a morte: Uma perspective psico-espiritual em cuidados paliativos domiciliares. O Mundo da Saúde, 27, 153-158.

Higginson, I., \& Priest, P. (1996). Predictors of family anxiety in the weeks before bereavement. Social Science \& Medicine, 43, 1621-1625.

Hodgson, C., Higginson, I., McDonnel, M., \& Butters, E. (1997). Family anxiety in advanced cancer: A multicentre prospective study in Ireland. British Journal of Cancer, 76, 1211-1214.

Jones, R. D. (2001). Depression and anxiety in oncology: The oncologist's perspective. The Journal of Clinical Psychiatry, 62, 52-57.

Kaasa, S., \& Loge, J. H. (2003). Quality of life in palliative care: Principles and practice. Palliative Medicine, 17, 1120.

Kessler, S. (1989). Psychological aspects of genetic counseling: VI. A critical review of the literature dealing with education and reproduction. American Journal of Medical Genetics, 34, 340-353.

Klinkenberg, M., Willems, D. L., Van der Wal, G., \& Deeg, D. J. H. (2004). Symptom burden in the last week of life. Journal of Pain and Symptom Management, 27, 5-13.

Kolcaba K., \& Fox C. (1999). The effects of guided imagery on comfort of women with early breast cancer undergoing radiation therapy. Oncology Nursing Fórum, 26(1), 67-72

Kovács, M. J. (2008). Desenvolvimento da Tanatologia: Estudos sobre a morte e o morrer. Paidéia (Ribeirão Preto), 18, 457-468.

Kristjanson, L. J., Leis, A., Koop, P. M., Carrière, K. C., \& Mueller, B. (1997). Family member'care expectations, care perceptions and satisfaction with advanced cancer care: Results of a multi-site pilot study. Journal of Palliative Care, 13, 5-13.

Kurtz, M. E., Kurtz, J. C., Given, C. W., \& Given, B. (1995). Relationship of caregiver reactions and depression to cancer patients symptoms, functional states and depression: A longitudinal view. Social Science \& Medicine, 40, 837-846

Lobchuk, M. M., \& Vorauer, J. D. (2003). Family caregiver perspective-taking and accuracy in estimating cancer patient symptom experiences. Social Science \& Medicine, $57,2379-2384$.

Nijboer, C., Triemstra, M., Tempelaar, R., Sanderman, R., \& van den Bos, G. A. (1999). Measuring both negative and positive reactions to giving care to cancer patiens: Psychometric qualities of the Caregiver Reaction Assessment (CRA). Social Science \& Medicine, 48, 1259-1269.

Novak, B., Kolcaba, K., Steiner, R., \& Dowd, T. (2001). Measuring comfort in caregiver and patients during late end-of-life care. The American Journal of Hospice \& Palliative Care, 18, 170-180.

Osse, B. H. P., Vernoij-Dassen, M. J. F. J., Vree, B. P. W., Schadé, E., \& Grol, R. P. T. M. (2000). Assessment of the need for palliative care as perceived by individual cancer patients and their families. Cancer, 88, 900-911. 
Parkes, C. M. (1998). Luto: Estudos sobre a perda na vida adulta (M. H. F. Bromberg, Trad.). São Paulo: Summus. (Original publicado em 1996)

Perdicaris, A. A. M. (2005). Além do bisturi: Novas fronteiras na comunicação médica. Santos, SP: Editora Universitária Leopoldianum.

Rabow, M. W., Hauser, J. M., \& Adams, J. (2004). Supporting family caregivers at the end of life: "They don't know what they don't know". JAMA: The Journal of The American Medical Association, 291, 483-491.

Rezende, V. L., Derchain, S. M., Botega, N. J., \& Vial, D. L. (2005). Revisão crítica dos instrumentos utilizados para avaliar aspectos emocionais, físicos e sociais do cuidador de pacientes com câncer na fase terminal da doença. Revista Brasileira de Cancerologia, 51, 79-87.

SAS Institute Inc. (1999-2001). SAS/STAT software changes and enhancements thougt release 8.2. Cary, NC: SAS Institute, Inc.

Silva, A. L. P. (2003). O acompanhamento psicológico a familiares de pacientes oncológicos terminais no cotidiano hospitalar. Interação em Psicologia, 7, 27-35.

World Health Organization. (1990). Cancer pain relief and palliative care. Geneva: WHO.

Zigmond, A. S., \& Snaith, R. P. (1983). The Hospital Anxiety and Depression Scale. Acta Psychiatrica Scandinavica, 67, 361-370.

Vera Lucia Rezende é Doutora em Tocoginecologia pelo Programa de Pós-graduação da Faculdade de Ciências Médicas, Centro de Atenção Integral à Saúde da Mulher, da Universidade de Campinas.

Sophie Derchain é Professora Associada do Departamento de Tocoginecologia da Faculdade de Ciências Médicas, Centro de Atenção Integral à Saúde da Mulher, da Universidade Estadual de Campinas.

Neury José Botega é Professor Titular do Departamento de Psicologia Médica e Psiquiatria, Faculdade de Ciências Médicas, Universidade Estadual de Campinas.

Luis Otávio Sarian é Professor Associado do Departamento de Tocoginecologia, Faculdade de Ciências Médicas, Centro de Atenção Integral à Saúde da Mulher, Universidade Estadual de Campinas.

Daniela Landulfo Vial é Psicóloga, com Aprimoramento em Psicologia em Saúde Reprodutiva da Mulher, Centro de Atenção Integral à Saúde da Mulher, Universidade Estadual de Campinas.

Sirlei Siani Morais é Estatística do Centro de Atenção Integral à Saúde da Mulher, Universidade Estadual de Campinas.

Antonio André Magoulas Perdicaris é Professor Titular da Faculdade de Medicina da Universidade Metropolitana de Santos, campus Constituição. 